Case Report

\title{
Strongyloides Colitis as a Harmful Mimicker of Inflammatory Bowel Disease
}

\author{
Julio Poveda, ${ }^{1}$ Farah El-Sharkawy, ${ }^{1}$ Leopoldo R. Arosemena, ${ }^{2}$ \\ Monica T. Garcia-Buitrago, ${ }^{1}$ and Claudia P. Rojas ${ }^{1}$ \\ ${ }^{1}$ Department of Pathology, University of Miami Health System/Jackson Memorial Hospital, 1611 NW 12th Ave, Holtz Center 2142D, \\ Miami, FL 33136, USA \\ ${ }^{2}$ Department of Gastroenterology, University of Miami Health System/Jackson Memorial Hospital, 1475 NW 12th Ave, Miami, \\ FL 33136, USA
}

Correspondence should be addressed to Claudia P. Rojas; crojas@med.miami.edu

Received 12 January 2017; Accepted 18 April 2017; Published 7 May 2017

Academic Editor: Dimosthenis Miliaras

Copyright @ 2017 Julio Poveda et al. This is an open access article distributed under the Creative Commons Attribution License, which permits unrestricted use, distribution, and reproduction in any medium, provided the original work is properly cited.

\begin{abstract}
Autoinfection caused by Strongyloides stercoralis frequently becomes a life-long disease unless it is effectively treated. There is overlapping histomorphology between Strongyloides colitis and inflammatory bowel disease; a low index of suspicion can lead to misdiagnosis and fatal consequences. We present a case of Strongyloides colitis mimicking the clinical and pathologic features of inflammatory bowel disease. A 64-year-old female presented to the emergency department with a four-day history of abdominal pain, diarrhea, and hematochezia. Colonoscopy revealed diffuse inflammation suggestive of inflammatory bowel disease, which led to initiation of 5-aminosalicylic acid and intravenous methylprednisolone. Biopsies of the colon revealed increased lymphoplasmacytic infiltrate of the lamina propria with eosinophilic microabscesses and presence of larvae, consistent with Strongyloides stercoralis. Immunosuppressive medication was halted. The patient ultimately died a few days later. This case emphasizes the importance of identifying the overlapping clinical and pathologic features of Strongyloides colitis and inflammatory bowel disease. A high index of suspicion and recognition of particular histological findings, including eosinophilic microabscesses, aid in the correct diagnosis. Definitive diagnosis is crucial as each disease carries distinct therapeutic implications and outcome.
\end{abstract}

\section{Introduction}

Strongyloidiasis is an infectious disorder caused by the nematode Strongyloides stercoralis, which is endemic in subtropical and tropical regions with poor sanitary conditions. In the United States, this infection is prevalent in several rural areas of the southeast and Appalachian region. The condition may be asymptomatic in immunocompetent patients, or it may manifest with occasional stomachache, intermittent diarrhea and constipation, bloating, nausea, and loss of appetite. Severe life-threatening complications of hyperinfection syndrome and disseminated strongyloidiasis may develop in patients with HTLV-1 confection or in patients receiving immunosuppressive therapy, such as corticosteroids [1-3].

\section{Case Report}

A 64-year-old Hispanic immigrant woman presented to the emergency department complaining of abdominal pain, hematochezia, and episodes of diarrhea that began four days prior to admission. She had a significant past medical history of diabetes mellitus, hypertension, rheumatoid arthritis, and diverticulosis. The patient had a general healthy appearance and was in no acute distress. Initial lab results reported a high leucocyte count $\left(16,000 / \mathrm{mm}^{3}\right)$, eosinophilia $(9.3 \%)$, anemia with hemoglobin of $9.9 \mathrm{~g} / \mathrm{dL}$, and hematocrit of $31.4 \%$.

The colonoscopy revealed inflammation characterized by congestion, edema, erythema, friability, and aphthous and confluent ulcerations throughout the entire colon (Figures 1 and 2). As these findings were suggestive of ulcerative colitis, 


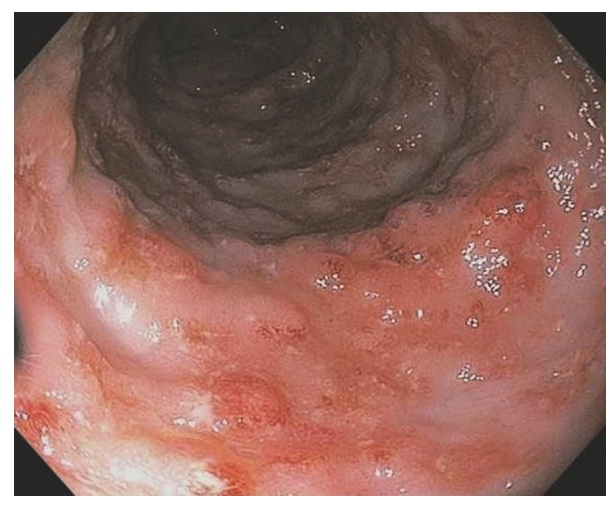

FIGURE 1: Colonoscopy showing portion of ascending colon with congested mucosa and aphthous and confluent ulcerations.

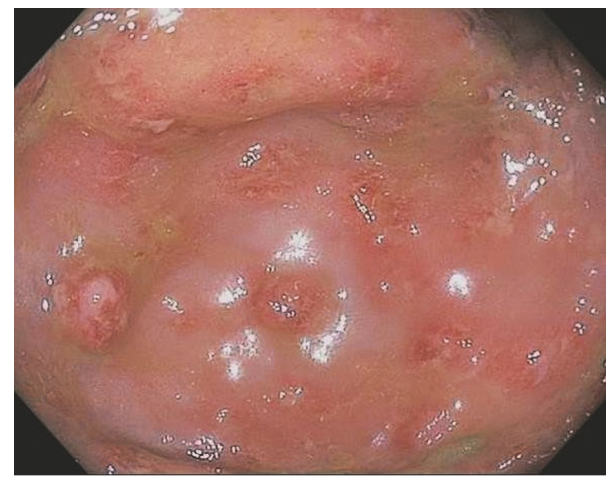

FIGURE 2: Colonoscopy showing portion of cecum with multiple aphthous ulcers.

a combination of oral 5-aminosalicylic acid and intravenous methylprednisolone was started. After two days of therapy, the patient suddenly became lethargic, tachycardic, and hypotensive. She was intubated for airway protection and was transferred to the intensive care unit.

Blood cultures returned positive for Gram-negative rods. Biopsies from colonoscopy revealed lymphoplasmacytic infiltrate of the lamina propria with mild architectural distortion, eosinophilic microabscesses, crypt abscesses, and presence of larvae, representative of Strongyloides infection (Figures 3-5). A diagnosis of strongyloidiasis and sepsis was made. Treatment with vancomycin, meropenem, metronidazole, fluconazole, valacyclovir, and ivermectin was initiated, and immunosuppressive therapy was halted. The patient was maintained on vasopressors and aggressive fluid hydration due to hemodynamic instability. The patient subsequently died due to the systemic complications of strongyloidiasis.

\section{Discussion}

Strongyloides stercoralis is unique in its ability to exist and replicate within a host for decades while remaining asymptomatic, or producing minimal nonspecific symptoms, until it transitions to a potentially fatal disseminated infection. Risk

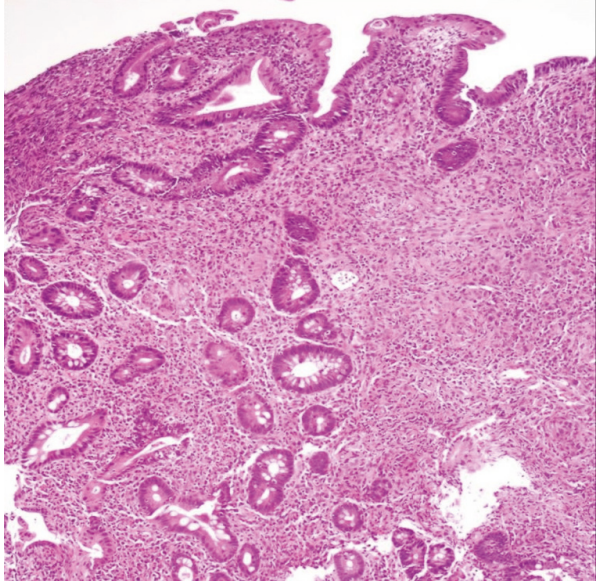

FIGURE 3: Colonic mucosa showing marked architectural distortion and crypt branching, increased lymphoplasmacytic and eosinophilic infiltrate in the lamina propria, and cryptitis. H\&E 10x.

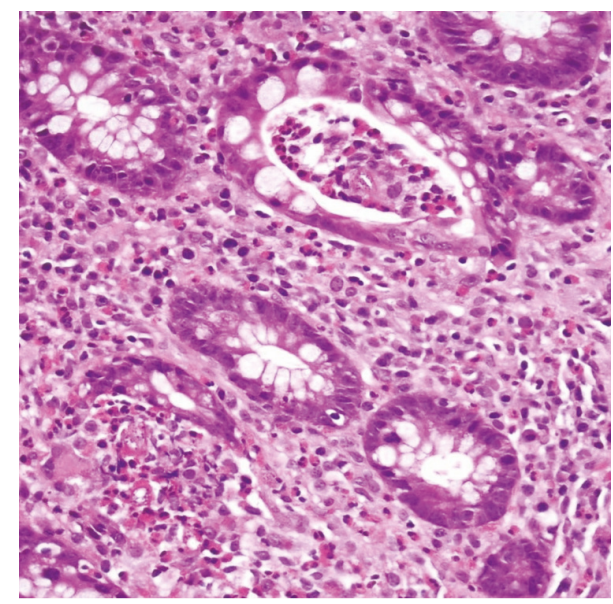

FIgURE 4: Eosinophilic microabscesses within glands and lymphoplasmacytic infiltrate in the lamina propria. H\&E 20x.

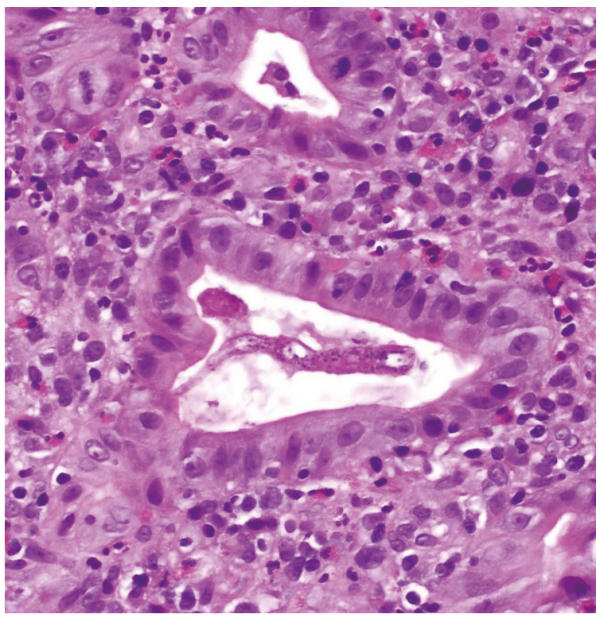

FIGURE 5: Strongyloides larva in the lumen of a crypt. H\&E 40x. 
factors to these complications are immunosuppression, corticosteroid therapy, transplantation, malnutrition, alcoholism, and HTLV-1 coinfection [1-6]. HTLV-1 coinfection is a particularly strong risk factor for severe forms of strongyloidiasis due to an impaired Th2 immune response $[1,5,6]$.

Infection occurs via penetration of larvae into the skin or mucous membranes from soil or feces. Once in the tissue, larvae enter the circulation and migrate into the alveolar spaces. The larvae ascend through the respiratory tract and are swallowed by the host, which leads them to the gastrointestinal tract. In the small bowel, larvae mature into adult females, which produce eggs through parthenogenesis. The excretion of larvae constitutes the mainstay of diagnosis via laboratory examination of stool $[1,2]$.

A unique characteristic of $S$. stercoralis is its ability to maintain an autoinfective cycle. Larvae reenter the circulation by invading the intestinal mucosa, or they may penetrate the perianal skin. Autoinfection occurs in hosts with an impaired cell-mediated immune response. The immunocompromised state allows for the development of the most severe forms of strongyloidiasis: hyperinfection syndrome and disseminated strongyloidiasis [1-3]. The mortality associated with these conditions can be as high as $87 \%$ [7].

Hyperinfection syndrome is a severe complication of longstanding infection in which there is an excessive increase in the worm load within the host. The most common risk factor is corticosteroid therapy $[3,7]$. Disseminated strongyloidiasis is characterized by abundant widespread larvae to extraintestinal sites, such as the lungs, heart, kidneys, central nervous system, and endocrine organs [4]. In the course of severe disseminated disease or hyperinfection, a patient may test positive for enteric or Gram-negative bacteremia due to the translocation of gut bacteria through an ulcerated mucosa $[2,8]$. Our patient suffered from sepsis as a complication of hyperinfection syndrome and disseminated strongyloidiasis following immunosuppressive therapy.

The macroscopic findings of strongyloidiasis on endoscopy are frequently confused with those of ulcerative colitis or Crohn's disease. Upper endoscopy usually reveals hyperemic edematous duodenal mucosa, erythema, friability, and white villi in the duodenum. Colonoscopy may show mucosal edema, erosions, submucosal hemorrhage, and ulcerations, which alternate with portions of normal mucosa [8-10]. The distinction between strongyloidiasis and inflammatory bowel disease (IBD) is made microscopically; however, there are significant overlapping features between both entities. Histological examination in strongyloidiasis reveals edema and infiltration of the lamina propria by lymphocytes, plasma cells and eosinophils, blunted villi, cryptitis, and crypt hyperplasia, which are also common findings in IBD [8, $9,11]$. In contrast to ulcerative colitis, inflammation caused by Strongyloides almost always extends into the submucosa and can be transmural; it often skips some areas in the involved segments (skip lesions), rarely involves the rectum, and shows milder crypt architecture distortion. Attenuation of the disease in the distal colon and rectum is common in strongyloidiasis, while ulcerative colitis characteristically affects the distal colon and rectum [11]. The skip lesions and eosinophilic granulomas found in strongyloidiasis are also found in Crohn's disease. However, the transmural granulomatous inflammatory process in strongyloidiasis is caused by the presence of larvae, a pathognomonic finding $[11,12]$. The granulomas often have abundant histiocytes or may have mature giant cells containing the remains of larvae [12]. Another distinctive feature is the formation of eosinophilic microabscesses in the lamina propria and submucosa [11].

Treatment for $S$. stercoralis colitis is drastically different from that for IBD. Antihelminthic therapy with ivermectin and withdrawal of immunosuppressive therapy are the treatments of choice for Strongyloides colitis [11]. In contrast, IBD is treated with corticosteroids, which has been proven to be the leading risk factor for the most severe forms of strongyloidiasis [1, 2, 4]. Thus, correct diagnosis of Strongyloides colitis is fundamental for the selection of an appropriate treatment. Misdiagnosis of IBD is common due to the similarities in clinical presentation, endoscopy, and histology. Attention to subtle morphologic differences between strongyloidiasis and IBD is critical for minimizing diagnostic error. This neglected condition requires a high index of suspicion, especially in patients infected with HTLV1 or who are undergoing treatment with corticosteroids or chemotherapy. Initiation of antihelminthic therapy for Strongyloides colitis and withholding immunosuppressive medications are crucial for preventing a fatal outcome in this curable disease.

\section{Disclosure}

This case study was presented at a poster session (poster no. 51) at the College of American Pathologists 2015 Annual Meeting, Nashville, Tennessee.

\section{Conflicts of Interest}

The authors declare that they have no conflicts of interest.

\section{References}

[1] E. M. Carvalho and A. Da Fonseca Porto, "Epidemiological and clinical interaction between HTLV-1 and Strongyloides stercoralis," Parasite Immunology, vol. 26, no. 11-12, pp. 487-497, 2004.

[2] R. Concha, W. Harrington Jr., and A. I. Rogers, "Intestinal strongyloidiasis: recognition, management, and determinants of outcome," Journal of Clinical Gastroenterology, vol. 39, no. 3, pp. 203-211, 2005.

[3] D. R. Boulware, W. M. Stauffer, B. R. Hendel-Paterson et al., "Maltreatment of Strongyloides infection: case series and worldwide physicians-in-training survey," American Journal of Medicine, vol. 120, no. 6, pp. 545.el-545.e8, 2007.

[4] P. B. Keiser and T. B. Nutman, "Strongyloides stercoralis in the immunocompromised population," Clinical Microbiology Reviews, vol. 17, no. 1, pp. 208-217, 2004.

[5] L. A. Marcos, A. Terashima, M. Canales, and E. Gotuzzo, "Update on strongyloidiasis in the immunocompromised host," Current Infectious Disease Reports, vol. 13, no. 1, pp. 35-46, 2011. 
[6] L. Fernandez, A. Gonzalez, L. F. Sua, D. Vargas, and C. A. Muñoz, "Síndrome de hiperinfección por Strongyloides stercoralis: diagnóstico por medio de citología de lavado broncoalveolar," Revista Colombiana de Neumología, vol. 27, pp. 318-327, 2015.

[7] R. S. Vadlamudi, D. S. Chi, and G. Krishnaswamy, "Intestinal strongyloidiasis and hyperinfection syndrome," Clinical and Molecular Allergy, vol. 4, article 8, 2006.

[8] R. Romero-Cabello, J. V. Gómez, M. H. González, and R. R. Feregrino, "Hyperinfection with Strongyloides stercoralis," BMJ Case Reports, 2012.

[9] B. F. Thompson, L. C. Fry, C. D. Wells et al., "The spectrum of GI strongyloidiasis: an endoscopic-pathologic study," Gastrointestinal Endoscopy, vol. 59, no. 7, pp. 906-910, 2004.

[10] K. Kishimoto, A. Hokama, T. Hirata et al., "Endoscopic and histopathological study on the duodenum of Strongyloides stercoralis hyperinfection," World Journal of Gastroenterology, vol. 14, no. 11, pp. 1768-1773, 2008.

[11] Z. Qu, U. R. Kundu, R. A. Abadeer, and A. Wanger, "Strongyloides colitis is a lethal mimic of ulcerative colitis: the key morphologic differential diagnosis," Human Pathology, vol. 40, no. 4, pp. 572-577, 2009.

[12] Y. Gutierrez, P. Bhatia, S. T. Garbadawala, J. R. Dobson, T. M. Wallace, and T. E. Carey, "Strongyloides stercoralis eosinophilic granulomatous enterocolitis," American Journal of Surgical Pathology, vol. 20, no. 5, pp. 603-612, 1996. 


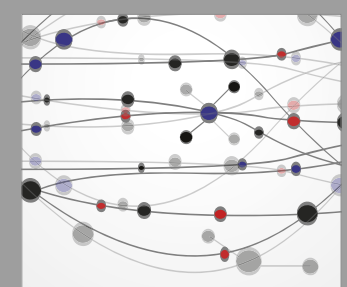

The Scientific World Journal
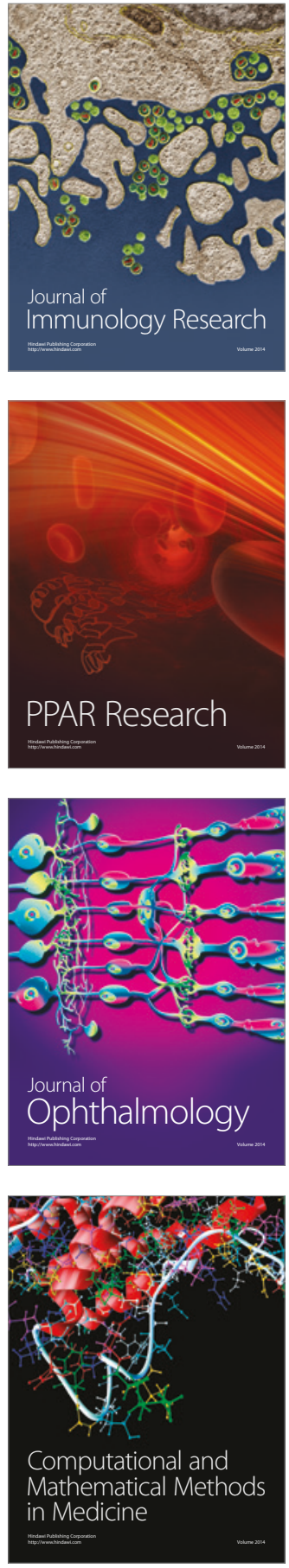

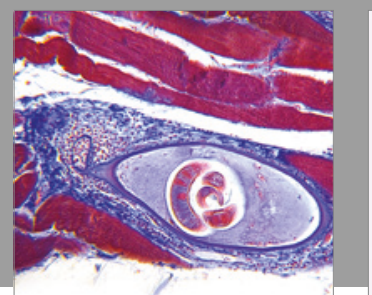

Gastroenterology Research and Practice
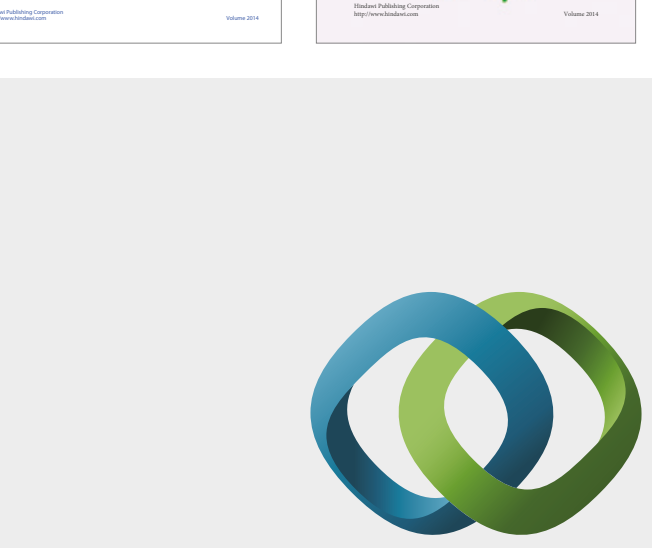

\section{Hindawi}

Submit your manuscripts at

https://www.hindawi.com
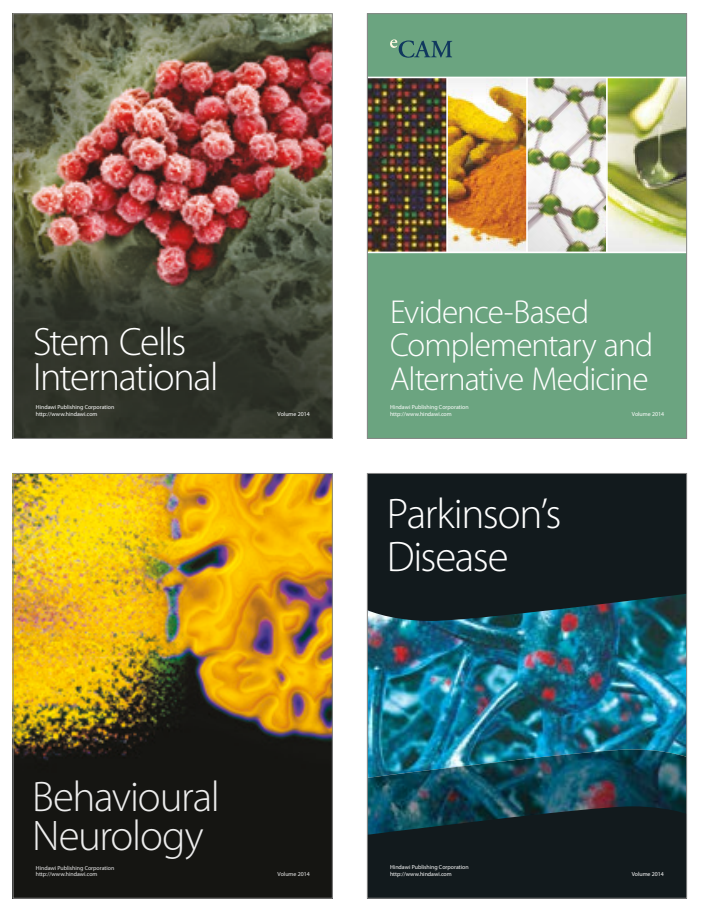
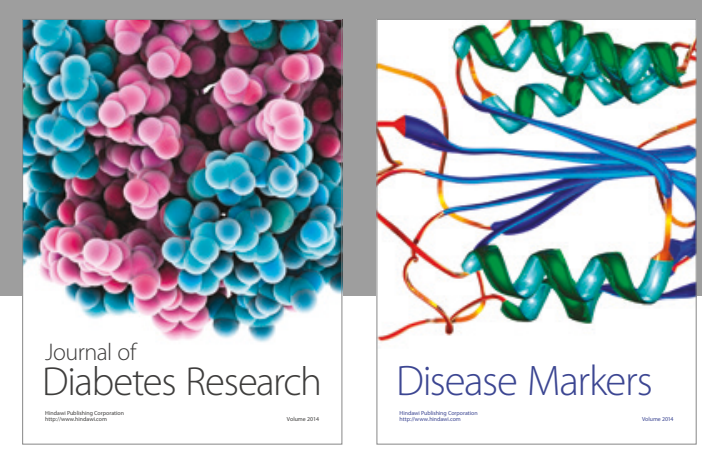

Disease Markers
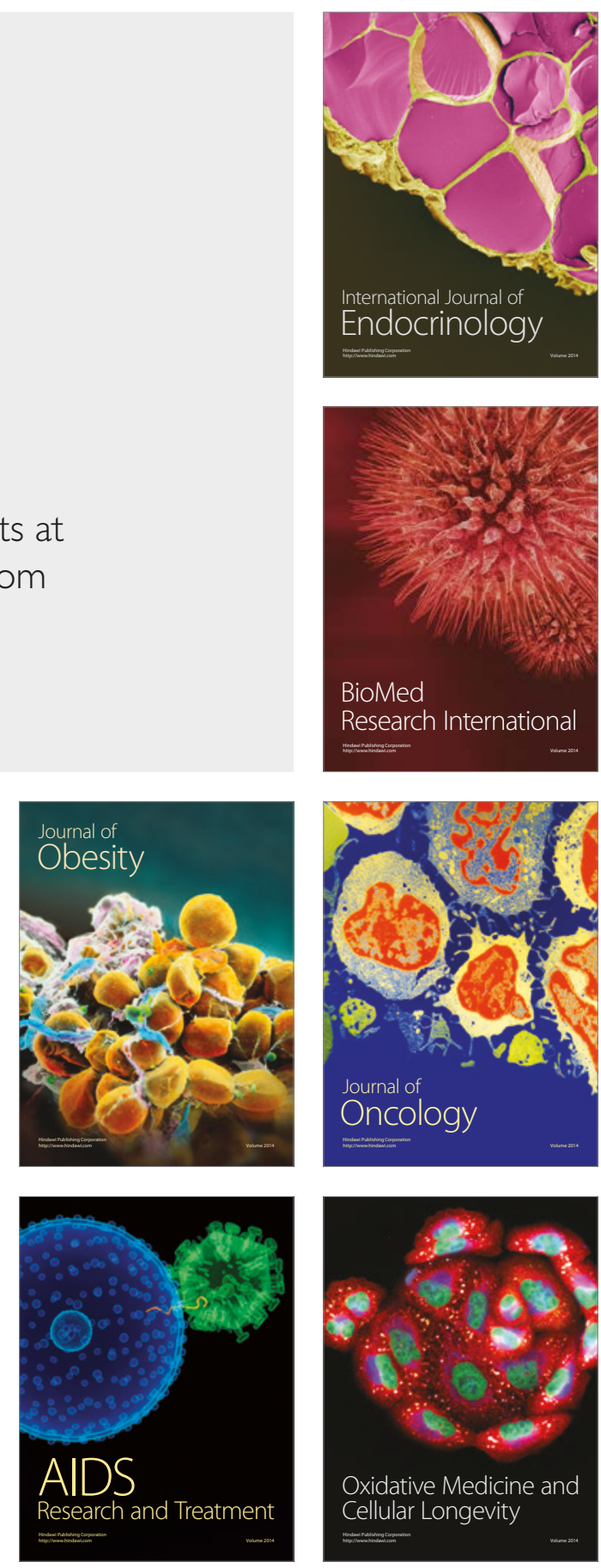